\title{
Ku80 Counters Oxidative Stress-Induced DNA Damage and Cataract Formation in the Human Lens
}

\author{
Andrew John Oliver Smith, ${ }^{1}$ Simon Sidney Robert Ball, ${ }^{1}$ Kamal Manzar, ${ }^{2}$ Richard Peter Bowater, ${ }^{1}$ \\ and Ian Michael Wormstone ${ }^{1}$
}

${ }^{1}$ School of Biological Sciences, University of East Anglia, Norwich Research Park, Norwich, United Kingdom

${ }^{2}$ Norwich Medical School, University of East Anglia, Norwich Research Park, Norwich, United Kingdom

Correspondence: Richard Peter Bowater, School of Biological Sciences, University of East Anglia, Norwich Research Park, Norwich, UK, NR4 $7 \mathrm{TJ}$;

r.bowater@uea.ac.uk

Ian Michael Wormstone, School of Biological Sciences, University of East Anglia, Norwich Research Park, Norwich, UK, NR4 7TJ;

i.m.wormstone@uea.ac.uk.

Submitted: October 1, 2015

Accepted: November 3, 2015

Citation: Smith AJO, Ball SSR, Manzar K, Bowater RP, Wormstone IM. Ku80 counters oxidative stress-induced DNA damage and cataract formation in the human lens. Invest Ophthalmol Vis Sci. 2015;56:7868-7874. DOI:10.1167/iovs.15-18309
Purpose. Oxidative stress in the human lens leads to a wide range of damage including DNA strand breaks, which are likely to contribute to cataract formation. The protein Ku80 is a fundamental component of the nonhomologous end-joining pathway that repairs DNA double strand breaks. This study investigates the putative impact of Ku80 in cataract prevention in the human lens.

Methods. The present study used the human lens epithelial cell line FHL124 and whole human lens organ culture. Targeted siRNA was used to deplete Ku80, with Western blot and immunocytochemistry employed to assess Ku80 expression levels. Oxidative stress was induced with hydrogen peroxide and DNA strand breaks measured by alkaline comet assay and $\gamma \mathrm{H} 2 \mathrm{AX}$ foci counts. Visual quality of whole human lenses was measured with image analysis software.

Results. Expression of Ku80 was predominately found in the cell nucleus of both FHL124 cells and native human lens epithelium. Treatment of FHL124 cells and whole lens cultures with siRNA targeted against Ku80 resulted in a significant knockdown at the protein level. Application of oxidative stress $\left(30 \mu \mathrm{M} \mathrm{H}_{2} \mathrm{O}_{2}\right)$ created more DNA strand breaks when added to Ku80 knockdown cells than in scrambled siRNA control cells as determined by the alkaline comet assay and the number of $\gamma \mathrm{H} 2 \mathrm{AX}$ foci. In whole lens cultures, exposure to $1 \mathrm{mM} \mathrm{H}_{2} \mathrm{O}_{2}$ resulted in more lens opacity in Ku80 knockdown lenses than match-paired controls.

Conclusions. Depletion of Ku80 in the lens through acute change or a consequence of aging is likely to increase levels of DNA strand breaks, which could negatively influence physiological function and promote lens opacity. It is therefore feasible that Ku80 plays a role in retarding cataract formation.

Keywords: lens epithelium, lens opacity, cataractogenesis, oxidative damage, DNA damage
$\mathrm{T}$ he genomes of all cells are continually damaged in many ways, by many agents. Such damage can disrupt normal cellular metabolism, leading to mutations and potentially cell death, and all cells have a complex of networks to detect and correct damage to essential molecules, such as DNA. For example, nonhomologous end-joining (NHEJ) repairs doublestrand breaks in the DNA backbone. ${ }^{1-3}$ There have been a number of studies investigating the cellular role(s) of NHEJ in human cells, but many questions remain unanswered, particularly in relation to its effects on human disease. ${ }^{4,5}$ Accumulated DNA damage is also associated with natural aging phenomena. ${ }^{2,6}$ Currently, the understanding of how such processes impact human physiology is incomplete, partly due to a lack of experimental models that link biochemical and cellular studies to tissue and/or organs.

The human lens serves as a valuable experimental model that permits investigation of physiological events at the cell and organ level ${ }^{7}$ (also see Royal Society podcast link, in the public domain: https://www.youtube.com/watch? $=8 \mathrm{q} 40 \mathrm{ZkIj} 81 \mathrm{w}){ }^{8}$ The lens is also affected by one of the major aging disorders, namely cataract. Cataract is defined as any opacity of the eye lens and is the leading cause of worldwide blindness; it largely afflicts the elderly and, presently, the only treatment is surgical intervention, ${ }^{9,10}$ which places significant burden on healthcare budgets. ${ }^{11,12}$ Delaying the onset of cataract is a major health care priority throughout the world.

Throughout life, the lens epithelium is exposed to potential DNA damaging stimuli. An important factor with respect to DNA damage and cataract formation is oxidative stress, ${ }^{13}$ which can result from reactive oxygen species generated during cellular metabolism or environmental exposure to acute or chronic levels of UV or ionizing radiation. ${ }^{14}$ Oxidative stress is known to induce in DNA both single strand breaks and double strand breaks. ${ }^{13,15,16}$ It is important to note that the lens epithelium is essential for maintenance of a healthy, transparent lens and is responsible for the production of antioxidants, which protect the lens fiber cells that form the central bulk of the lens. ${ }^{17}$ The viability of the lens epithelium has therefore been suggested to be important in maintaining lens transparency, with apoptosis demonstrated to be increased in cataract lenses. ${ }^{18}$ It is conceivable that apoptotic events may be prompted by unrepaired DNA damage, such as strand breaks.

Few studies to date have assessed the impact of DNA damage in the lens epithelium or upon cataract formation $^{13,19,20}$ and any roles of specific DNA repair pathways have not been identified. The present study detected and examined 
the role of Ku80, a core protein in NHEJ of DNA double strand breaks. ${ }^{3-6,21}$ The protein Ku80 associates with Ku70 to form a heterodimer that binds DNA double strand breaks. The complex Ku70/80 engages several other proteins that process the DNA damage and coordinate its repair. Nonhomologous end-joining is considered the major mammalian pathway involved in the repair of DNA double strand breaks and, unlike homologous recombination, acts throughout the cell cycle ${ }^{1}$; this is of particular relevance to the lens when one considers that a large proportion of the epithelium is nondividing.

To clarify potential links between oxidative stress, DNA damage and physiological function of the human lens, we examined the role of Ku80 in this complex sequence of events. To investigate this, we employed a combination of a human lens epithelial cell line (FHL124) ${ }^{13,22-24}$ and whole human lens organ culture. ${ }^{23}$ Oxidative damage acts to impair function of the human lens, which promotes the onset of cataract formation. This study provides the first data showing that Ku80 plays an important role in retarding this process in the human lens. Thus, the maintenance of an active NHEJ repair system will facilitate healthy aging of the human lens and stave off cataract formation.

\section{Materials AND Methods}

\section{Cell Culture}

The human lens epithelial cell line FHL124 is a nonvirally transformed cell line generated from human capsule-epithelial explants (Refs. 13, 22-24; Reddan JR, et al. IOVS 1999;40:ARVO Abstract 5110). This cell line demonstrates a $99.5 \%$ homology to native lens epithelium in transcript profile. ${ }^{22}$ We cultured FHL124 cells at $35^{\circ} \mathrm{C}$ with $5 \% \mathrm{CO}_{2}, 95 \%$ air in Eagle's minimum essential medium (EMEM; Gibco, Paisley, UK) supplemented with 5\% vol/vol fetal calf serum (FCS; Gibco) and $50 \mu \mathrm{g} / \mathrm{mL}$ gentamicin (Sigma-Aldrich Corp., Dorset, UK). We seeded FHL124 cells onto 96-well plates, 35-mm tissue culture dishes $(35,000 /$ dish for alkaline comet assay and Western blot) or $18 \times 18$ glass coverslips contained within $35-\mathrm{mm}$ tissue culture dishes (10,000/coverslip for immunocytochemistry). Culture medium was replaced with EMEM without FCS, 24 hours prior to start of experimental conditions.

\section{Whole Human Lens Culture}

Donor eyes were obtained from the East Anglian Eye Bank with written informed consent obtained from next of kin. The research was approved by the UK National Research Ethics Committee (REC 04/Q0102/57) and followed the tenets of the Declaration of Helsinki regarding the use of human donor material. Following removal of corneas for transplant, lenses were dissected by anterior approach and cultured within 24 hours post mortem as previously described. ${ }^{23}$ Briefly, following dissection, lenses were maintained in EMEM supplemented with $50 \mu \mathrm{g} / \mathrm{mL}$ gentamicin at $35^{\circ} \mathrm{C}, 5 \% \mathrm{CO}_{2}, 95 \%$ air for 24 hours prior to addition of experimental conditions to ensure no damage had arisen during dissection.

\section{Knockdown of Ku80 Expression}

Expression of Ku80 was depleted in both FHL124 cells and whole human lens cultures using siRNA (Qiagen, Crawley, UK) against the target sequence (5'-AAGCATAACTATGAGTGTTTA$\left.3^{\prime}\right)$ with controls transfected with control siRNA (AllStars Negative, scramble siRNA; Qiagen) using oligofectamine transfection reagent (Invitrogen, Carlsbad, CA, USA). All dilutions and subsequent culture was performed in Opti-
MEM. FHL124 cells were transfected with siRNA at $5 \mathrm{nM}$ for 48 hours, whole human lens cultures with siRNA at $10 \mathrm{nM}$ for 72 hours. Following transfection periods FHL124 cells were either lysed for protein analysis by Western blot or placed in experimental conditions and subsequently analyzed for DNA strand breaks by alkaline comet assay. Following the transfection period for whole human lenses, in some cases the epithelium was isolated by dissection and fixed with $4 \%$ formaldehyde for immunocytochemistry, lysed for protein analysis or preparations were placed in experimental conditions to allow lens opacity to be evaluated.

\section{Immunocytochemistry}

Coverslips or tissue samples were fixed with $4 \%$ vol/vol formaldehyde (Sigma-Aldrich Corp.) in PBS for 30 minutes followed by three washes in PBS for 5 minutes. Cells or tissue were permeabilized with $0.5 \%$ vol/vol Triton X-100 (SigmaAldrich Corp.) for 30 minutes. Three washes in PBS containing $0.02 \% \mathrm{wt} / \mathrm{vol} \mathrm{BSA}$ and $0.05 \% \mathrm{vol} / \mathrm{vol}$ commercial detergent (IGEPAL; Sigma-Aldrich Corp.) were performed before blocking for nonspecific binding sites with normal donkey serum (1:50 in 1\% wt/vol BSA in PBS) for 1 hour. Ku80 or $\gamma \mathrm{H} 2 \mathrm{AX}$ primary antibody (Cell Signaling Technology, Danvers, MA, USA) was diluted (1:100 or 1:200 respectively in 1\% BSA in PBS) and applied overnight at $4{ }^{\circ} \mathrm{C}$. Three further washes in PBS containing $0.02 \% \mathrm{wt} / \mathrm{vol} \mathrm{BSA}$ and $0.05 \% \mathrm{vol} / \mathrm{vol}$ IGEPAL were performed followed by addition of secondary antibody (AlexaFluor 488-conjugated donkey anti-rabbit (Invitrogen) diluted 1:100 in $1 \%$ BSA in PBS) for 1 hour, protected from light at $37^{\circ} \mathrm{C}$ in a humidified atmosphere. Coverslips or tissue samples were then counterstained with $4^{\prime}$,6-diamidino-2-phenylindole (DAPI; Sigma-Aldrich Corp.) and Texas red-x-phalloidin (Invitrogen). A further three washes in $0.02 \% \mathrm{wt} / \mathrm{vol} \mathrm{BSA}$ and $0.05 \% \mathrm{vol} / \mathrm{vol}$ IGEPAL were performed followed by mounting of samples onto glass microscope slides. Samples were viewed with fluorescence microscopy (widefield microscope Zeiss AxioPlan 2ie; Carl Zeiss Microscopy GmbH, Gottingen, Germany) and images captured with a digital camera and microscopy software (AxioVision; Carl Zeiss Microscopy Ltd., Cambridge, UK). Image quantification was performed using ImageJ $1.45 \mathrm{~s}$ image analysis software (http://imagej.nih.gov/ij/; provided in the public domain by the National Institutes of Health, Bethesda, MD, USA).

\section{Western Blot Analysis}

Cell lysates from FHL124 cells were prepared using M-PER buffer (Thermo Fisher Scientific, Basingstoke, UK) supplemented with phosphatase and protease inhibitors and $0.5 \mathrm{M}$ EDTA (Thermo Fisher Scientific) at $10 \mu \mathrm{L} / \mathrm{ml}$ immediately prior to use. Total protein content was determined by the BCA assay (Pierce, Thermo Fisher Scientific) to enable loading of equal amounts of protein per sample onto $10 \%$ SDS-polyacrylamide gels. Proteins were transferred to polyvinylidene fluoride membrane using a semidry transfer cell. Membranes were blocked with PBS containing 5\% wt/vol nonfat dry milk and $0.1 \% \mathrm{vol} / \mathrm{vol}$ Tween-20, hybridized with primary antibody (Cell Signaling Technology), followed by incubation with secondary antibody (GE Healthcare, Little Chalfont, UK). Antibodies bound to proteins were detected using a Western blotting detection system (ECL Plus; GE Healthcare).

\section{Alkaline Comet Assay}

To assess levels of DNA strand breaks in FHL124 cells, the alkaline comet assay was performed. Briefly, following experimental treatments, cells were washed with ice cold 

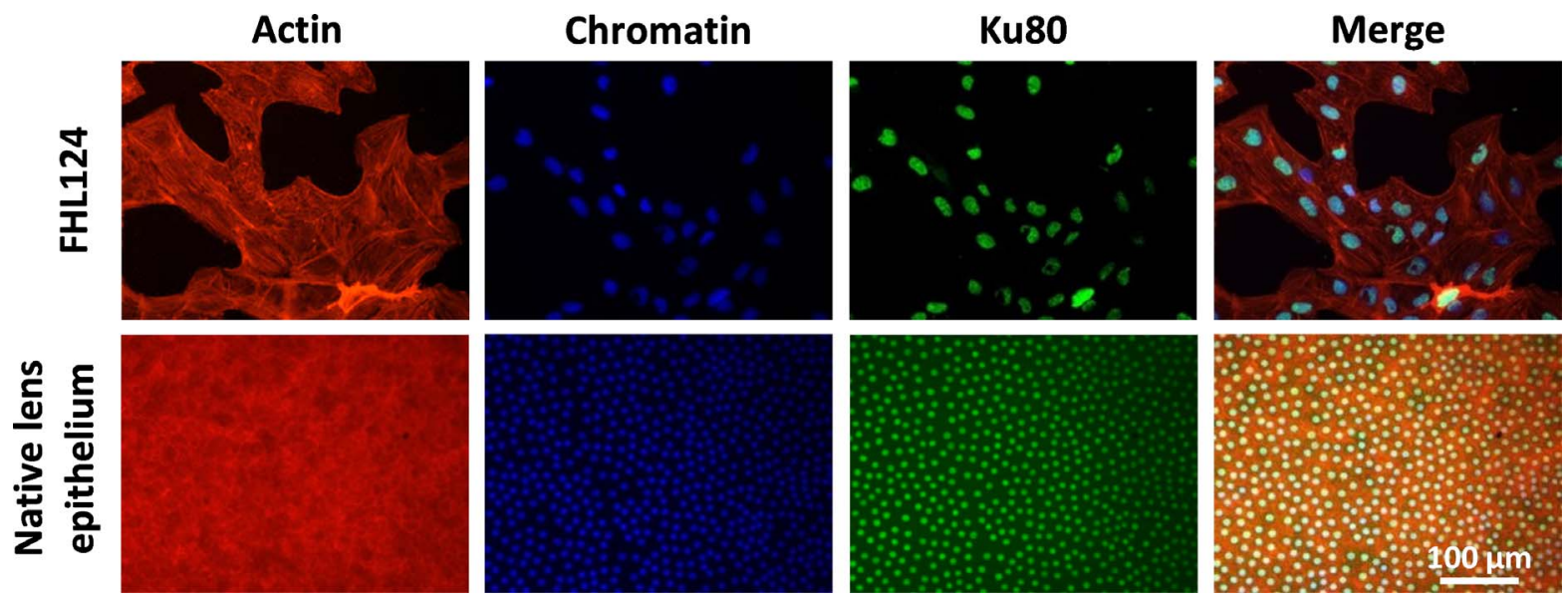

FIGURE 1. Fluorescent micrographs showing Ku80 expression in FHL124 cells and native human lens epithelium. Visualization of antibody to Ku80 was with AlexaFluor 488-conjugated secondary antibody; actin was visualized by Texas Red-X Phalloidin; and chromatin by DAPI stain. The data are representative of at least three preparations examined.

PBS, harvested, counted, resuspended in PBS containing 10\% dimethyl sulfoxide and frozen at $-80^{\circ} \mathrm{C}$ until the alkaline comet assay was performed as previously described. ${ }^{13}$

\section{Analysis of Human Lenses for Reduction in Visual Quality}

Lenses were imaged using a charge-coupled device (CCD) camera (UVP, Cambridge, UK) equipped with commercial software (Synoptics, Cambridge, UK) at the experimental start point (day 0) and at 24-hour intervals throughout experiments. Brightfield illumination was used, with a black and white grid placed beneath lenses to assess visual quality and, therefore, lens clarity. Visual quality was quantified from these images by measuring standard deviation values of grayscale values obtained from the grid beneath the lens. Values of standard deviation of clear lenses are high, whereas when the lens becomes more opaque, the grid becomes less defined/ homogenous and the standard deviation values decrease. A reference value for peak visual quality was determined for each image by selecting a region of the grid adjacent to the lens. This region exhibits the greatest standard deviation and best visual quality and thus a decrease was calculated relative to these values. Image analysis was performed with analysis software (Image-Pro Premier; Media Cybernetics, Inc., Rockville, MD, USA).

\section{Statistical Analysis}

One-way ANOVA with post hoc Tukey's test was performed to determine statistical differences between multiple experimental groups (SPSS 16.0; SPSS, Inc., Chicago, IL, USA) and Student's $t$-test to determine statistical differences between two experimental groups (Microsoft Excel; Microsoft Corp., Redmond, WA, USA). A $P$ value of $\leq 0.05$ was considered significant.
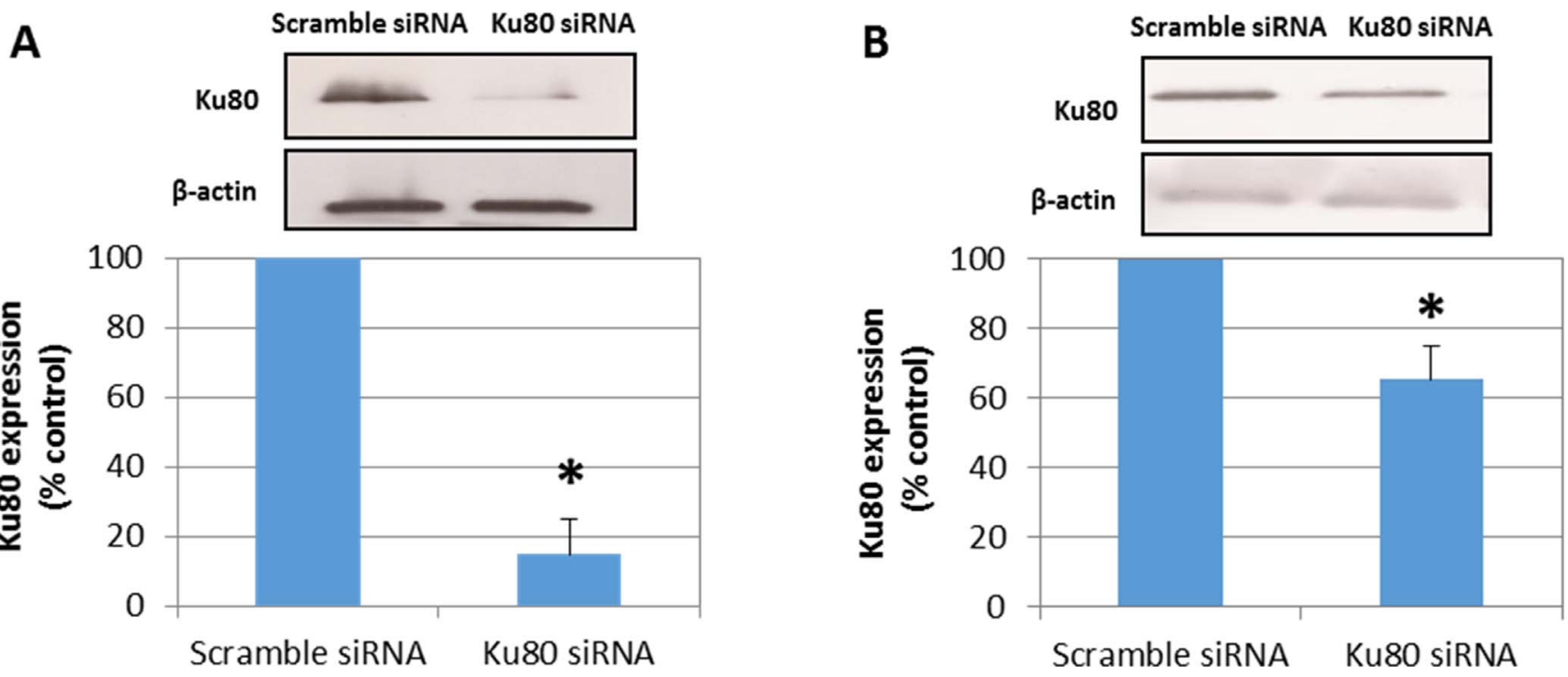

Figure 2. Validation of Ku80 knockdown in human lens epithelial cells. Depletion of Ku80 by targeted siRNA in (A) FHL124 cells and (B) whole human lenses relative to control scramble siRNA treatment detected using Western blot methods. Cells were exposed to siRNA treatments for 48 hours (FHL124 cells) or 72 hours (whole lens cultures). The data presented show representative blots and quantitative data from three separate experiments in each case. The graphs are presented as mean \pm SEM. *Significant difference between groups (Student's $t$-test; $P \leq 0.05$ ). 
A

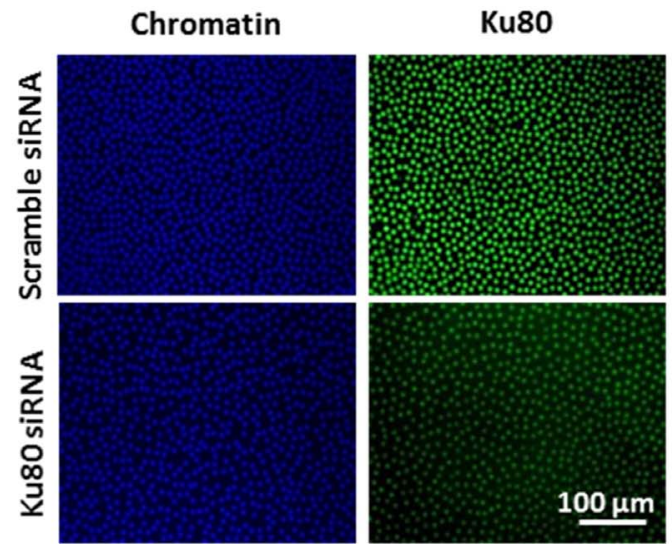

B

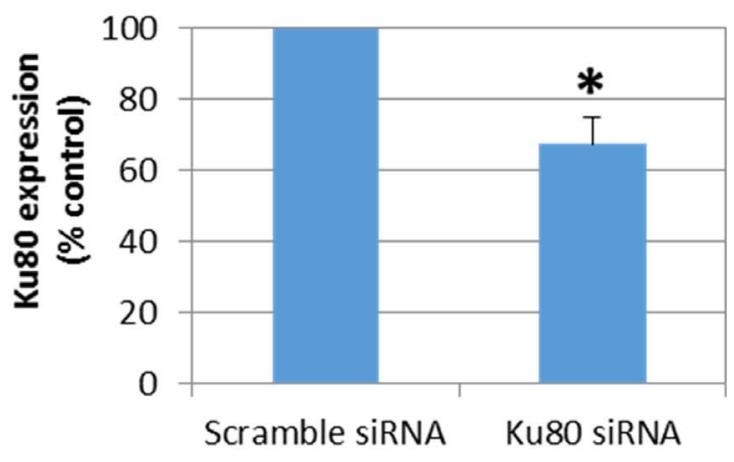

FIgURE 3. Depletion of Ku80 by targeted siRNA in the epithelium of cultured whole human lenses relative to scramble controls. (A) Representative fluorescent micrographs and (B) quantitative analysis of nuclear Ku80 levels. Antibody to Ku80 was visualized with an AlexaFluor 488 conjugated secondary antibody and chromatin by DAPI stain. The quantitative data presented is derived from four separate experiments and are presented as mean \pm SEM. *Significant difference between groups (Student's $t$-test; $P \leq 0.05$ ).

\section{Results}

Oxidative stress is a known causative factor of cataract formation in the human lens, but the relevance of DNA damage on this physiological effect remains unclear. To extend knowledge of this complex situation, we studied Ku80, an important component of the NHEJ DNA repair pathway, beginning by analyzing its expression in FHL124 cells and human lenses by immunocytochemistry. In both FHL124 cells and the native epithelium, Ku80 was detected predominantly in the cell nucleus (Fig. 1), which is consistent with its expected function in NHEJ. To ascertain the effects of depleted Ku80 expression on lens cell function, a targeted siRNA approach was adopted. Expression of Ku80 was significantly depleted in FHL124 cells following a 48-hour treatment period, such that an $85.7 \% \pm 7.6 \%$ knockdown was achieved

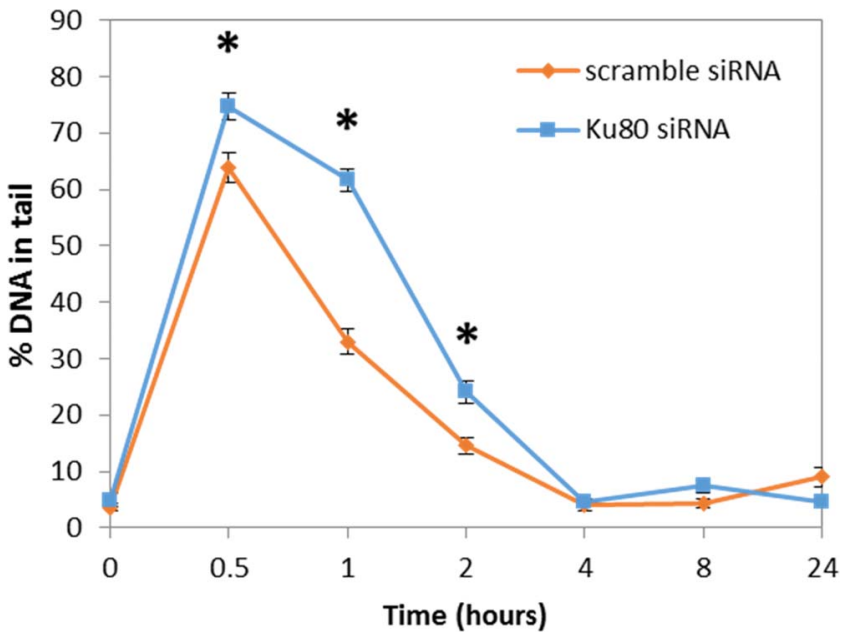

Figure 4. Depletion Ku80 increases $\mathrm{H}_{2} \mathrm{O}_{2}$-induced DNA strand breaks in FHL124 cells. Cells were treated with siRNA targeted against Ku80 or scramble control siRNA. Breaks of DNA strands were measured by the alkaline comet assay. Cells were exposed to $30 \mu \mathrm{M} \mathrm{H}_{2} \mathrm{O}_{2}$ to induce an oxidative stress. The data presented is representative of three separate experiments. The data are presented as mean $\pm \mathrm{SD} .{ }^{*}$ Indicates a significant difference between targeted and control siRNA treatment groups (ANOVA with post hoc Tukey test $P \leq 0.05$ ) compared with cells treated with noncoding scrambled siRNA (Fig. 2A). No obvious phenotypical changes in cells treated with either Ku80 siRNA or noncoding scrambled control siRNA were noted at this stage. With respect to whole lens cultures, levels of Ku80 were assessed using Western blot (Fig. 2B) and immunocytochemistry (Fig. 3) following a 72-hour transfection period. The overall expression in the Ku80 siRNA-treated group of whole lenses was significantly lower than controls treated with noncoding scrambled siRNA.

In order to test the effect of depleted Ku80 expression on lens cell susceptibility to oxidative stress-induced DNA strand breaks, FHL124 cells with Ku80 expression depleted with targeted siRNA were treated with $\mathrm{H}_{2} \mathrm{O}_{2}$. Previous work using the comet assay ${ }^{13}$ reported that $30 \mu \mathrm{M} \mathrm{H}_{2} \mathrm{O}_{2}$ can induce DNA strand breaks in FHL124 cells and thus this concentration was used in the current study. Peak levels of DNA strand breaks were observed in Ku80 knockdown cells at 0.5 hours post treatment $(74.8 \% \pm 2.4 \%$ DNA in tail). These levels were significantly greater than those observed at 0.5 hours post treatment in scrambled siRNA treated control cells (61.6 \pm $2.1 \%$ DNA in tail) and remained significantly elevated until the 4-hour time point (Fig. 4).

Since Ku80 is an important component of NHEJ that repairs DNA double strand breaks, we specifically assessed the level of double strand breaks by determining $\gamma \mathrm{H} 2 \mathrm{AX}$ foci in FHL124 cells (Fig. 5). Knockdown Ku80 cells increased foci number relative to scrambled siRNA control cells, but this difference was not significant. Treatment with $\mathrm{H}_{2} \mathrm{O}_{2}$ resulted in a significant increase of foci numbers in Ku80 knockdown cells relative to scrambled siRNA controls.

To assess the effect of Ku80 depletion on the sensitivity of human lenses to oxidative stress, $1 \mathrm{mM} \mathrm{H}_{2} \mathrm{O}_{2}$ was applied followed by a 1-day culture period. A moderate loss in transparency appeared in the central region of the lens cortex when transfected with noncoding scrambled siRNA at day 1 compared to day 0 following $\mathrm{H}_{2} \mathrm{O}_{2}$ treatment. However, a marked decrease in visual quality in Ku80 knockdown lenses was clearly evident at day 1 compared with day 0 . In this case, a greater degree of the lens cortex was affected and, at 24 hours, a significant decrease in visual quality was observed in Ku80 knockdown lenses compared with their match-paired controls (transfected with noncoding scrambled control siRNA; Fig. 6). 
A
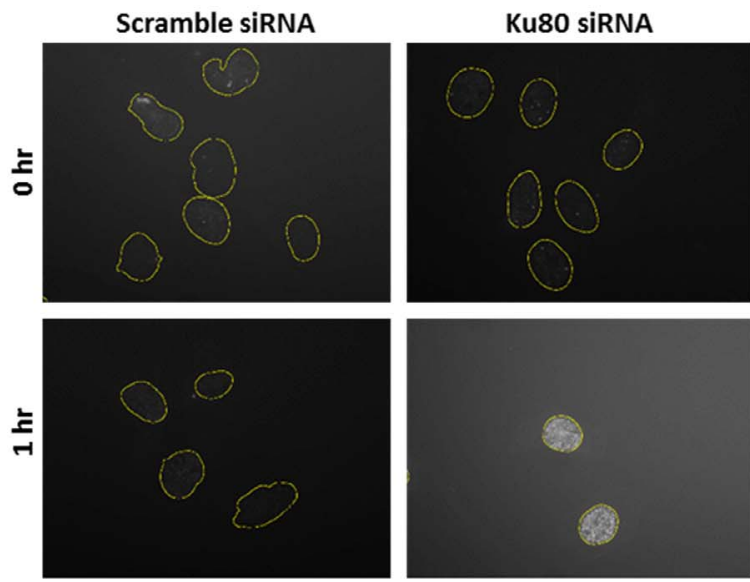

B

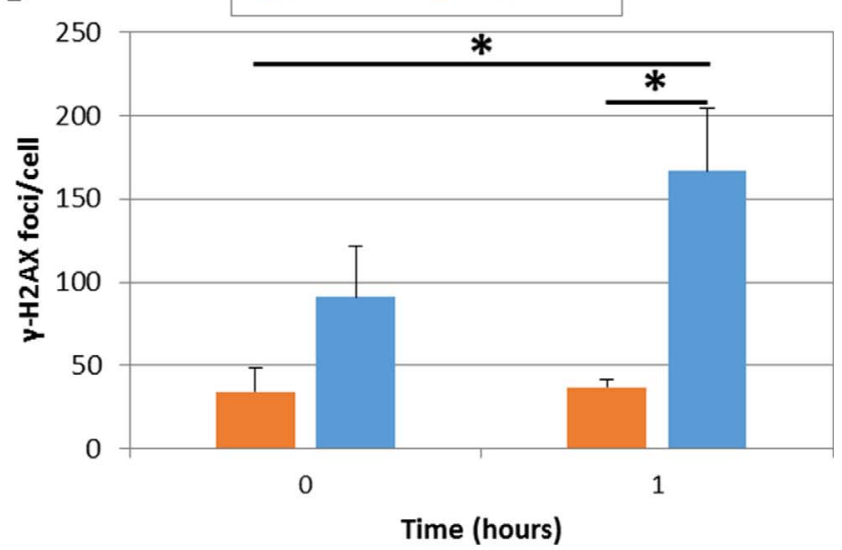

Figure 5. Oxidative stress increases DNA double strand breaks in Ku80 depleted FHL124 cells. $\gamma \mathrm{H} 2 \mathrm{AX}$ was used as a measure of DNA double strand breaks, such that foci per cell nucleus (defined by a yellow outline) were determined. (A) Representative fluorescent micrographs and (B) quantitative analysis. The data are presented as mean \pm SEM $(n=3)$. *Significant difference between groups (ANOVA with post hoc Tukey's test; $P \leq 0.05$ )

\section{Discussion}

It is well established that DNA damage and DNA repair processes are fundamental to the health of human cells and tissues and accumulated DNA damage is also associated with natural aging phenomena. ${ }^{1-3,6}$ However, how such processes impact on human physiology is not fully understood. With this in mind, we have developed the human lens as an experimental model that allows biochemical and cellular studies to be linked directly to observations in related tissue and/or organs. ${ }^{13,22-24}$

The current study has shown that siRNA can be used to deplete Ku80 expression in the whole human lens, albeit to a lesser extent than observed in FHL124 cells. When the lenses were exposed to $\mathrm{H}_{2} \mathrm{O}_{2}$, depletion of $\mathrm{Ku} 80$ expression rendered them more susceptible to oxidative stress-induced changes in opacity compared with their match-paired controls, presumably due to the increased levels of DNA damage leading to loss of cell function or death. Impaired cell function in the epithelium could disrupt many cellular processes, which is likely to impact on overall ionic regulation within the lens. This in turn could lead to elevation of calcium within fiber cells, which is typical of cortical cataract, and activation of calcium dependent proteases, such as calpains. ${ }^{25}$

Cataract is an age-related disease and is associated with oxidative stress. ${ }^{14,26}$ As humans age, the ability of the lens to cope with oxidative stress declines; reduced glutathione, a crucial lens antioxidant is diminished ${ }^{27,28}$ and, thus, lens fibers become more susceptible to oxidative damage. Since the lens epithelium produces the antioxidants such as reduced glutathione, damage to the epithelial cells, including DNA damage, may result in further reductions of antioxidant defense molecules. This could diminish cellular function and have a detrimental effect on the transparency of the fiber mass, which is reliant on the epithelium for support and maintenance. With aging, this general decrease in the ability of the lens to cope with oxidative insult renders the lens increasingly vulnerable to oxidative damage and cataract. Should a further failing in DNA repair pathways occur, such as decreased repair by NHEJ, for example, this susceptibility to

B

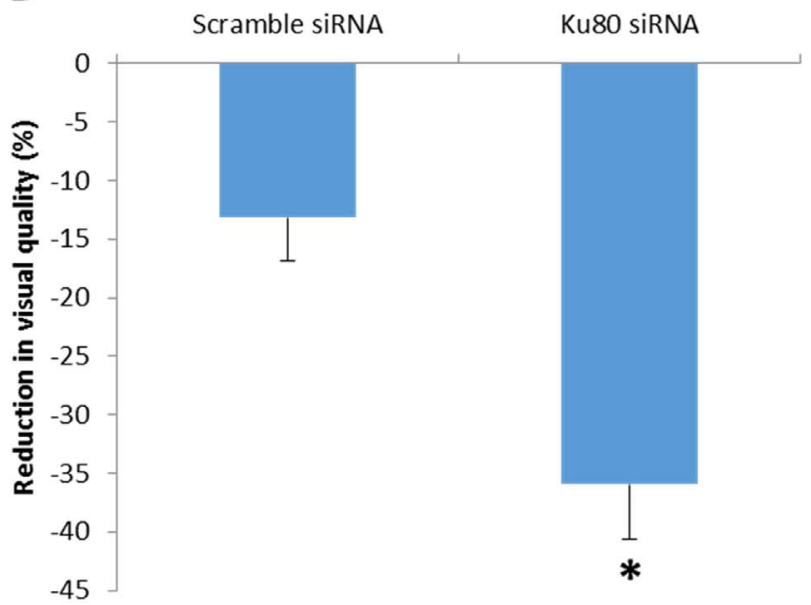


oxidative damage or a loss of epithelial cell function could be further exacerbated.

The ability of human cells to cope with DNA damage also declines with age, which has been linked to an accumulation of mutations resulting from a decline in DNA damage repair systems such as NHEJ. ${ }^{29}$ A core protein of NHEJ, Ku80 has been implicated in the aging process. Seluanov and colleagues ${ }^{30}$ observed that the level of Ku80 (and Ku70) declines during cellular senescence. Vogel et al. ${ }^{31}$ observed that a deletion of Ku80 in mice causes early onset of age-specific changes such as osteopenia, atrophic skin and hepatocellular degeneration; they were also reported to have a shorter lifespan. They did not, however, report any observations of the lenses of the mice. Cataract is a disease closely associated with aging; therefore, a measure of lens opacity within Ku80 negative mice would provide an excellent additional measure of aging within that experimental system.

In the case of the human lens, a suppression of Ku80 was observed rather than total ablation. However, this still generated distinct physiological outcomes, such that lenses were indeed more susceptible to oxidative stress-induced opacity. In fact, this type of effect is likely to be more physiologically relevant since Ku80 (and other DNA repair proteins) are likely to decline gradually with age rather than face total depletion of protein expression. Cataract is primarily an age-related disease and, therefore, a declining function in how aged lenses cope with oxidative stress and its resulting DNA damage may be crucial in the formation of the disease. In eukaryotic cells, NHEJ accounts for the majority of DNA double strand break repair and, if double strand breaks go unrepaired, they can result in cell death. ${ }^{32}$ Apoptosis in the lens epithelial cell population has been linked to cataract formation. ${ }^{18}$ Cell death in the lens epithelium as a consequence of DNA damage may impair protection of the lens fibers against oxidative damage, ${ }^{17,33}$ which could promote the onset of cataract formation. The experimental model now provides a platform on which to identify the mechanistic regulation of NHEJ (and other DNA repair systems) with human cells and tissue.

In summary, functional disruption of the lens epithelium, as a consequence of DNA damage, may impair protection of the lens fibers against oxidative damage, ${ }^{17,33}$ which in turn could promote the onset of cataract formation in the human lens. The data presented suggest Ku80 plays an important role in retarding this process and maintaining an active NHEJ repair system will facilitate healthy aging ${ }^{3,6}$ and delay cataract formation.

\section{Acknowledgments}

The authors thank John Reddan for providing the FHL124 cell line, and Sarah Russell, Julie Eldred, and Meghan Betts for assistance.

Supported by the Humane Research Trust (IMW, RPB).

Disclosure: A.J.O. Smith, None; S.S.R. Ball, None; K. Manzar, None; R.P. Bowater, None; I.M. Wormstone, None

\section{References}

1. Goodarzi AA, Jeggo PA. The repair and signaling responses to DNA double-strand breaks. Adv Genet. 2013;82:1-45.

2. Iyama T, Wilson DM III. DNA repair mechanisms in dividing and non-dividing cells. DNA Repair. 2013;12:620-636.

3. Jackson SP, Bartek J. The DNA-damage response in human biology and disease. Nature. 2009;461:1071-1078.

4. Radhakrishnan SK, Jette N, Lees-Miller SP. Non-homologous end joining: emerging themes and unanswered questions. DNA Repair. 2014;17:2-8.
5. Woodbine L, Gennery AR, Jeggo PA. The clinical impact of deficiency in DNA non-homologous end-joining. DNA Repair. 2014;16:84-96.

6. Hoeijmakers JH. DNA damage, aging, and cancer. $N$ Engl $J$ Med. 2009;361:1475-1485.

7. Wormstone IM, Wride MA. The ocular lens: a classic model for development, physiology and disease. Philos Trans $R$ Soc Lond B Biol Sci. 2011;366:1190-1192.

8. The Royal Society. The ocular lens: a classic model for development, physiology and disease [Video]. YouTube. https://www.youtube.com/watch?v=8q40ZkIj81w. Published March 30, 2011. Accessed September 22, 2015.

9. Wormstone IM, Wang L, Liu CS. Posterior capsule opacification. Exp Eye Res. 2009;88:257-269.

10. Allen D, Vasavada A. Cataract and surgery for cataract. BMJ. 2006;333:128-132.

11. Spalton DJ, Russell SL, Evans-Gowing R, Eldred JA, Wormstone IM. Effect of total lens epithelial cell destruction on intraocular lens fixation in the human capsular bag. J Cataract Refract Surg. 2014;40:306-312.

12. Brown GC, Brown MM, Menezes A, Busbee BG, Lieske HB, Lieske PA. Cataract surgery cost utility revisited in 2012: a new economic paradigm. Ophthalmology. 2013;120:2367-2376.

13. Liu H, Smith AJ, Lott MC, et al. Sulforaphane can protect lens cells against oxidative stress: implications for cataract prevention. Invest Ophthalmol Vis Sci. 2013;54:5236-5248.

14. Spector A. Oxidative stress-induced cataract: mechanism of action. FASEB J. 1995;9:1173-1182.

15. Sestili P, Cattabeni F, Cantoni O. Simultaneous determination of DNA double-strand breaks and DNA fragment size in culturedmammalian-cells exposed to hydrogen-peroxide histidine or etoposide with chef electrophoresis. Carcinogenesis. 1995; 16:703-706.

16. Collins AR. Measuring oxidative damage to DNA and its repair with the comet assay. Biochim Biophys Acta. 2014;1840:794800 .

17. Michael R, Bron AJ. The ageing lens and cataract: a model of normal and pathological ageing. Philos Trans $R$ Soc Lond $B$ Biol Sci. 2011;366:1278-1292.

18. Li WC, Kuszak JR, Dunn K, et al. Lens epithelial cell apoptosis appears to be a common cellular basis for non-congenital cataract development in humans and animals. J Cell Biol. 1995;130:169-181.

19. Kleiman NJ, Spector A. DNA single strand breaks in human lens epithelial cells from patients with cataract. Curr Eye Res. 1993;12:423-431.

20. Sorte K, Sune P, Bhake A, Shivkumar VB, Gangane N, Basak A. Quantitative assessment of DNA damage directly in lens epithelial cells from senile cataract patients. Mol Vis. 2011; 17:1-6.

21. Fell VL, Schild-Poulter C. The Ku heterodimer: function in DNA repair and beyond. Mutat Res Rev Mutat Res. 2015;763: 15-29.

22. Wormstone IM, Tamiya S, Eldred JA, et al. Characterisation of TGF-beta2 signalling and function in a human lens cell line. Exp Eye Res. 2004;78:705-714.

23. Wang L, Eldred JA, Sidaway P, et al. Sigma 1 receptor stimulation protects against oxidative damage through suppression of the ER stress responses in the human lens. Mech Ageing Dev. 2012;133:665-675.

24. Wormstone IM, Eldred JA. Experimental models for posterior capsule opacification research [published online ahead of print May 1, 2015]. Exp Eye Res. doi:10.1016/j.exer.2015.04. 021.

25. Sanderson J, Marcantonio JM, Duncan G. A human lens model of cortical cataract: $\mathrm{Ca} 2+$-induced protein loss, vimentin 
cleavage and opacification. Invest Ophthalmol Vis Sci. 2000; 41:2255-2261.

26. Truscott RJ. Age-related nuclear cataract-oxidation is the key. Exp Eye Res. 2005;80:709-725.

27. Giblin FJ. Glutathione: a vital lens antioxidant. J Ocul Pharmacol Ther. 2000;16:121-135.

28. Harding JJ. Free and protein-bound glutathione in normal and cataractous human lenses. Biochem J. 1970;117:957-960.

29. Gorbunova V, Seluanov A. Making ends meet in old age: DSB repair and aging. Mech Ageing Dev. 2005;126:621-628.
30. Seluanov A, Danek J, Hause N, Gorbunova V. Changes in the level and distribution of $\mathrm{Ku}$ proteins during cellular senescence. DNA Repair. 2007;6:1740-1748.

31. Vogel H, Lim DS, Karsenty G, Finegold M, Hasty P. Deletion of Ku86 causes early onset of senescence in mice. Proc Natl Acad Sci U S A. 1999;96:10770-10775.

32. Jeggo PA, Geuting V, Lobrich M. The role of homologous recombination in radiation-induced double-strand break repair. Radiother Oncol. 2011;101:7-12.

33. Lou MF. Redox regulation in the lens. Prog Retin Eye Res. 2003;22:657-682. 